\title{
Hemorrhoidal Hemorrhage, CTCAE
}

National Cancer Institute

\section{Source}

National Cancer Institute. Hemorrhoidal Hemorrhage, CTCAE. NCI Thesaurus. Code C143537.

A disorder characterized by bleeding from the hemorrhoids. 